\title{
Device Exposure Domain
}

National Cancer Institute

\section{Source}

National Cancer Institute. Device Exposure Domain. NCI Thesaurus. Code C102617.

A subject domain utilized for the submission of information encompassing and representing data, vocabulary or records related to device exposure. 